\title{
Using HMD Virtual Reality to investigate individual differences in visual processing styles
}

Savickaite, S. ${ }^{1 *}$, McNaughton, K. ${ }^{1}$, Gaillard, E. ${ }^{1}$, Amaya, J. ${ }^{1}$, McDonnell, N. ${ }^{2}$, Millington, E. ${ }^{1}$ \& Simmons, D.R. ${ }^{1}$

\footnotetext{
${ }^{1}$ School of Psychology, University of Glasgow, Glasgow, Scotland, UK

${ }^{2}$ School of Humanities, University of Glasgow, Glasgow, Scotland, UK
}

\begin{abstract}
Global and local processing is part of human perceptual organisation, where global processing enables us to extract the 'gist' of the visual information and local processing helps us to perceive the details. Individual differences in these two types of visual processing have been found in autism and ADHD. Virtual Reality (VR) has become a more available method of research in the last few decades. No previous research has investigated perceptual differences using this technology. The standard ROCF test was used as a baseline task to look at a practical aspect of using VR as an experimental platform. 94 participants were tested. Attention-to-Detail, Attention Switching and Imagination subscales of AQ questionnaire were found to be predictors of organisational ROCF scores, whereas only Attention-toDetail subscale was predictive of perceptual ROCF scores. Current study is an example of how classic psychological paradigms can be transferred into the virtual world. Further investigation of the distinct individual preferences in drawing tasks in VR could lead to a better understanding on how we process visuospatial information. As a result, such findings would inevitably extend to industrial applications.
\end{abstract}

Keywords: Virtual Reality, individual differences, autism, ADHD, Rey-Osterrieth Complex Figure

*Corresponding author. Contact: sarune.savickaite@glasgow.ac.uk 


\section{Introduction}

Our visual system collates many separate pieces of information to construct a meaningful perception of the world (Goldstein \& Brockmole, 2016). The ability to detect and interpret such emerging visual patterns is often called "perceptual organisation" (Wagemans et al, 2012). Individual experiences are private and inaccessible to experimenters, but there are distinct processing styles which can be investigated. For example, global processing is the ability to extract the 'gist', whereas local processing focuses on the detailed information of a visual scene (Simmons \& Todorova, 2018). We all combine local elements of perception to extract the overall meaning, however, how we use this information to understand the world around us varies. Individual differences in local and global processing between individuals have been observed as early as infancy (Freeseman, Colombo and Coldren, 1993). Gender (Roalf, Lowery and Turetsky, 2005; Sadler-Smith, 2011), race (McKone et al, 2010) and mood (Gasper and Clore, 2002) have also been found to influence our preferred processing style. These differences extend to neurodivergent populations, with distinct preferences being reported in Obsessive Compulsive Disorder (Yovel, Revelle and Mineka, 2005), schizophrenia (Johnson, Lowery, Kohler and Turetsky, 2005), eating disorders (Li et al, 2015; Lopez et al, 2008), autism (e.g. Kandalaft, Didehbani and Krawczyk, 2013) and ADHD (Wang and Reid, 2011).

\section{Autism}

Autism Spectrum Disorders (ASDs) ${ }^{2}$ are a group of neurodevelopmental conditions characterised by difficulties with social interactions, sensory processing and repetitive behaviour (APA, 2013). As currently diagnosed, the best estimates of the prevalence of ASD is approximately $1 \%$ in western industrialized populations like the UK (e.g. Baird et al, 2006; Brugha et al, 2011). Autism research has largely overlooked the sensory world experienced by autistics, rather focusing on understanding and supporting the behavioural symptoms related to the condition (Baron-Cohen, 2017). Despite this, there exists a substantial body of evidence that an atypical pattern of visual processing is present in autistic individuals (Fleischmann et al., 2012; Simmons et al., 2009) and it may even manifest in commonly recognised behavioural symptoms (Happé \& Ronald, 2008). Therefore, investigating how visual information is processed by autistics is essential if we wish to gain a fundamental understanding of their perceptual experience, and ultimately ensure more appropriate support is provided (Bogdashina, 2016).

Autistics have commonly been reported to experience hypo- (under-reactivity to sensory stimuli) and hyper-sensitivity ('overload' of sensory stimuli) in addition to "sensory seeking" behaviours

\footnotetext{
${ }^{2}$ ASD, autism and individuals with autism will be used interchangeably in this paper. Recent research on neurodiversity and person-first vs. identity-first language, suggests this is an appropriate use of terminology (see Bury, Jellett, Spoor and Hedley (2020)).
} 
(Bogdashina, 2005; Schauder \& Bennetto, 2016). Self-report accounts of autistic individuals clearly show that their perception of the world is different from that of neurotypicals (Grandin and Duffy, 2008; Fleischmann, 2012). 'Sensory overload' is often discussed as one of the key aspects of autistic perceptual experience, where everyday sensory stimulation accumulates and becomes overwhelming (Robertson and Simmons, 2015). 'Fragmented world' is another aspect of perception where autistic individuals report seeing the world as isolated fragments rather than the coherent whole picture apparently perceived by neurotypicals (Frith, 2003) .

Several theoretical frameworks have been formulated to explain these perceptual anomalies. The first discussion of atypical perceptual organisation in autism was described by Shah and Frith (1983). They proposed the Weak Central Coherence (WCC) theory (Frith, 1989; Shah and Frith, 1993) to explain the exceptional performance of autistic individuals in a variety of visuospatial tasks, such as the embedded figures task, block design and visual illusions (Happe and Frith, 1996). WCC suggests that autistic individuals have a superior local processing style, where details of the object are perceived immediately, and often 'pop out' from the context. Shah and Frith $(1983,1993)$ observed a local bias in children with autism and a failure to extract global information. These researchers thus proposed that local and global processing are independent and governed by two separate mechanisms.

The Enhanced Perceptual Functioning (EPF) theory was proposed by Mottron and colleagues in the early 2000s (Mottron and Burack, 2001; Mottron, Dawson, Soulieres, Hubert and Burack, 2006). EPF offers an alternative explanation where autistic individuals have enhanced low-level perception and global perception is optional. They observed a local preference in autistic participants and a lack of automated extraction of global information, thus suggesting that autistic participants favour, but are not limited to, a local processing style (Van der Hallen et al, 2015).

The Executive Dysfunction (EFD) theory, postulated by Ozonoff, Pennington \& Rogers (1991), relates to executive function difficulties in autism. Autistic individuals were found to perform differently from controls on several Executive Function (EF) tasks involving working memory and reasoning (Lai et al., 2017; Chen et al., 2016; Minshew et al., 1994). The Executive Dysfunction theory links atypical frontal lobe function to autism, by suggesting EF difficulties in autism may be linked to atypical development of the frontal lobes, potentially through synaptic connectivity, pruning or myelination (Hutsler \& Casanova, 2016; Hill, 2004). This was supported by Luna et al (2007), who identified fronto-parietal circuitry differences as a plausible cause for different Working Memory performance on neuropsychological tasks in autism. They also specified that, while controls seemed to reach prefrontal cortex maturity around 19 years old, autistic participants did not reach maturity until 25 years old. 
These theoretical frameworks have been investigated for many years; however, no unified consensus has been reached yet and research in this area remains highly controversial. Van Der Hallen et al's (2015) meta-analysis of 56 studies covering 1000 ASD participants suggests that the major problem is often the studies themselves. They often differ in terms of participant group composition, sample size, stimuli, and task demands. Moreover, the definition of local and global processing is often unclear (Happé and Booth, 2008; Simmons and Todorova, 2018). There is also evidence of publication bias and that the array of tests used might not be focusing on the correct aspects of perceptual organisation (Van der Hallen et al, 2015). It is also argued that autistic differences in visual processing, when compared to typically developing individuals, may have been exaggerated (Van der Hallen et al., 2015), and that many sensory difficulties are identifiable across a significant proportion of the general population (Robertson \& Simmons, 2013; Cribb et al., 2016). Overall, contradictory findings regarding local and global processing preferences for both autistic and non-autistic samples have yet to be resolved (Muth et al., 2014; Van der Hallen et al., 2015). Recently researchers have proposed a dimensional construct of autism (Fletcher-Watson \& Happe, 2019). This model suggests that autistic traits are distributed continuously across a spectrum in both autistic and non-autistic populations. Therefore, studying a general population is a practical way to gain further insight into the visual processing styles adopted by individuals expressing varying degrees of autistic traits.

\section{ADHD}

ADHD is a neurodevelopmental condition characterised by patterns of inattention, hyperactivity and impulsivity (APA, 2013). ADHD traits have also been observed in the general population (Lubke et al, 2009). Some of the symptoms of ADHD have been found to be similar to ASD (Panagiotidi, Overton and Stafford, 2018) with 30-50\% of ASD cases meeting ADHD criteria (Hoftman et al, 2009). DSM-5 (APA, 2013) allows the simultaneous diagnosis of both conditions on the basis that both share similar hereditary causes (Lichtenstein et al, 2010) and executive dysfunction (Rommelse et al, 2011). When ASD and ADHD co-occur they often interact. Antshel and Hier (2014) found that people with ADHD, who also have co-diagnosed ASD, have more severe ADHD symptoms. Individuals with ADHD have also been found to perform differently on global tasks (Cohen and Kolantrhogg, 2018, Song and Hakoda, 2015) thus following similar patterns to autistics.

According to the findings of Song \& Hakoda (2015), children with ADHD demonstrated a reduced preference towards global processing which contradicts DSM-5 (APA, 2015) criteria where failure to pay close attention to detail was emphasised. This finding was further supported by Cohen et al. (2019) and Kalanthroff, Naparstek and Henik (2013), suggesting that individuals with ADHD may experience a local processing bias. Currently, the DSM-5 reports that symptoms of inattention for individuals with ADHD may be a manifestation of their difficulty with fully attending and concentrating on small details of their perceptual environment (APA, 2013). This statement is 
contradictory to recent findings, suggesting that the diagnostic criteria for ADHD may require updating. Furthermore, these recent findings suggest that autism and ADHD may share similarities in their overall visual processing style that may explain the overlap of symptoms (e.g. behavioural issues, difficulties with social interaction, especially in a neurotypical context). Currently, the extent of such overlap remains unclear (Groom et al., 2017) and warrants further investigation.

\section{The Rey-Osterrieth Complex figure (ROCF) and autism/ADHD}

The Rey-Osterrieth Complex figure (ROCF) was designed by Andre Rey (1941) and formalized by Paul-Alexandre Osterrieth (1944) and was initially used in studies of neuropsychological cases, such as stroke or brain damage assessment (Figure 1). The figure is presented, and the participant is asked to copy it on a piece of paper. Different colours are often used at different time increments to identify which part of the picture participants draw first. The second part of the test investigates participants' ability to recall the figure. The copy can be made immediately, after a short delay or both. There are many scoring systems for the ROCF. In our study we will use the perceptual (Osterrieth, 1944) and organisational (Hamby, Wilkins and Barry, 1993) scoring systems.

ROCF is a complex task assessing visuospatial memory (Shin et al, 2014), planning (Mohtor et al, 2018), sensory processing style (Tsatsanis et al, 2011) and executive dysfunction (Watanabe et al, 2005). It allows investigation of the processing and organisation of visuospatial information and helps the evaluation of executive function (Wilson and Bachelor, 2015). All three drawing conditions require higher processing strategies, as organising the complex figure into a coherent and meaningful whole is a task that requires planning, organising, working memory and attention (Shin et al., 2014; Beebe et al., 2009). For the recall conditions, Shorr, Delis and Massman (1992) believe that the prefrontal cortex guides the ROCF task, as frontal lobe processes strategically encode, retrieve and store visuo-spatial information. Weber et al. (2013) add that evidence from reduced ROCF performance in individuals with neuropsychological conditions reflects the number of executive function processes linked to the task.

The ROCF task has been used to research individual differences in local and global processing in Obsessive Compulsive Disorder (Moritz and Wendt, 2006), epilepsy (McConley et al, 2006), Alzheimer's disease (Melrose, 2013), eating disorders (Lopez et al, 2008; Lang et al, 2016), autism (Kuschner, Bodner and Minshew, 2009) and ADHD (Barkley, Grodzinky and duPaul, 1992, Miller and Hinshaw, 2010). 


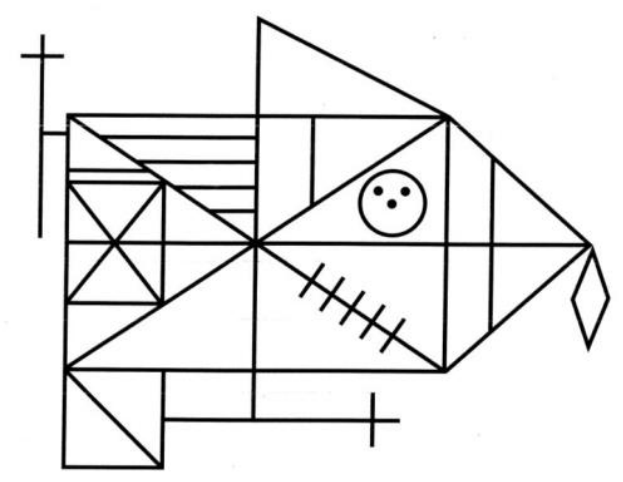

Figure 1. Rey- Osterrieth Complex Figure (ROCF) first described by Andre Rey in 1941 and later standardised by Paul-Alexandre Osterrieth in 1944

Developmental studies of the ROCF are of particular interest to the current studies, as both ASD and ADHD are neurodevelopmental conditions associated with delayed maturation of prefrontal cortex. Akshoomoff and Stiles (1995a, 1995b) provided valuable insight into developmental changes in ROCF tasks. They found that, before the age of 9 years old, children struggled to appreciate the figure as a coherent whole, instead adopting a piecemeal approach. This is similar to the method of processing proposed in WCC theory. The ROCF was used as a measure of executive function in studies with clinical groups of both ADHD and ASD. For instance, it was found that autistics were more likely to adopt a locally-oriented processing style than the non-autistic groups, and children with ADHD tended to show particular difficulties with the delayed recall condition (Van Eylen et al., 2018; Kiselev, 2019). Akshoomoff and Stiles (1995b) found that the strategy adopted in the copy condition influences the manner in which children will draw it from memory, and therefore their overall performance. The idea of drawing "styles" and "strategies" is intriguing and may provide additional insight into individual differences in cognitive processing. An attempt at evaluating trends in ROCF processing styles was initiated by Waber and Bernstein (1985), as they classified drawings into three categories: 'part-oriented', 'intermediate' and 'configurational' depending on drawing organisation.

\section{Virtual Reality (VR) and autism/ADHD}

Ever since the acquisition of Oculus by Facebook in 2014 (Dredge, 2014), Virtual Reality (VR) has come into focus as an available and useful tool for research. VR has been used in research since the late 1980s, however, this 2014 acquisition marked a new milestone for the technology. Equipment has become cheaper, more advanced and more readily available. VR now offers authenticity and a high level of realism as well as experimental control, thus facilitating learning and transfer of skills in the real world. As a result, Virtual Reality provides an experimental context with strong ecological validity (Parsons, 2016; Savickaite et al, 2021). 
In recent years, VR has been used in autism and ADHD research on social interaction training and intervention (for review see Savickaite et al, 2021). Social skills training has been performed with both autistic children (Matsentidou and Poullis, 2014) and adults (Kandaloft, Didehbani and Krawczyk, 2013; Bellani, Fornasari and Chittaro, 2011). Ke and Im (2013) constructed a virtual dolphinarium for autistic children and Smith et al (2014) developed an interview training set-up for autistic adults. These are just few examples of VR helping autistic people experience the world they are normally cautious of. Social and crowded situations are often a trigger for anxiety attacks in autism (Kuriakose \& Lahiri, 2016). With VR, autistic individuals can experience realistic scenarios without having to leave the safety of the room. Anxiety and phobia VR therapy in autism has proven to be successful and is now available via the UK National Health Service (Maskey, Lowry, Rodger, McConachie and Parr, 2014).

Vision scientists initially used VR cautiously to understand the human visual system due to several issues with the equipment (Paulus, Straube and Eggert, 2017; Elias, Batumalai and Azmi, 2019). With the technology leaping forward over the last few decades, most of these issues have been addressed and partially or completely resolved (Padmanaban, Konrad, Stramer, Cooper and Wetzstein, 2017; Laffont and Hasnain, 2017). It is now an ideal time to use virtual reality in research on human perceptual organisation.

\section{Current Experiment}

The current study is exploratory work as there are no previous studies on a similar subject. We will incorporate all of the elements discussed. Virtual Reality will be used to investigate individual differences in local and global processing styles. ASD and ADHD questionnaires will measure participants' individual differences. The Rey-Osterrieth Complex Figure task will be our tool to investigate these differences in the virtual environment.

We predict that the immersive aspects of VR will enhance differences between local and global processing. The direction of the difference is still debatable and due to the lack of studies on perception in VR we are unable to predict how the virtual environment might affect these interactions. The current study is also a baseline task to look at practical aspects of using TiltBrush and VR as an experimental platform.

\section{Methods}

\section{Participants}

Data was collected from January 2019 to June 2020 at the VR lab in the Philosophy Building, School of Humanities, University of Glasgow. Participants were recruited via Facebook post or word of mouth. Reward for participation was £6 per hour or course credit. 94 participants were tested, of whom $35(37.2 \%)$ were male and $46(62.8 \%)$ were female. The mean age of participants was 25.27 
years $(\mathrm{SD}=5.1)$. Participants were asked if they had any issues with their vision, and $36(38.3 \%)$ reported having some form of corrected vision. No serious visual impairments were reported. In addition, 37 (39.4\%) participants had experienced Virtual Reality prior to the experiment.

\section{Materials}

Participants were tested using an Nvidia GTX 1080 I Gaming PC (Windows 10) with HTC Vive Pro kit (Figure 2). The experiment was run through TiltBrush (Google, 2016) using SteamVR software (Steam, 2003).

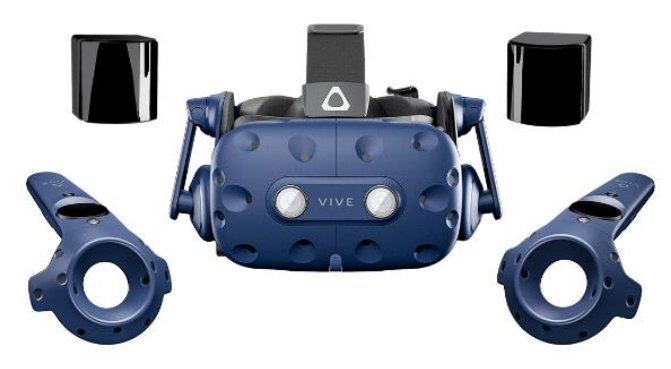

Figure 1. HTC Vive Pro kit (image from www.vive.com)

Questionnaire data was recorded using Microsoft Office Forms online using the same computer.

\section{Questionnaires}

\section{Technical Proficiency Questionnaire}

A questionnaire was devised for the study in order to gather additional information on participants' familiarity with VR, modern technology and collect information on any visual impairments they might have. The questionnaire was composed of 9 questions (available in online supplementary materials).

\section{Autism Spectrum Quotient Questionnaire}

The Autism Spectrum Quotient Questionnaire (AQ; Baron-Cohen, 2001) is a questionnaire devised to assess autistic traits in the general population. It comprises 50 items, 10 for each of 5 subscales: social skill, attention switching, attention-to-detail, communication and imagination (Baron Cohen et al, 2001). The questionnaire uses a 4-point agreement scale: definitely disagree, slightly disagree, slightly agree, definitely agree. Autistic traits are scored as 1 and non-autistic traits as 0 . Scores of 32+ are considered high and are often an indicator that the individual might have diagnosable ASD (AbuAkel, et al., 2019). The AQ has been found to be a good tool for investigating the continuum of autistic expression in the general population. Internal consistency and test-retest reliability of the questionnaire are largely consistent throughout different demographics and cultures (Stevenson and 
Hart, 2017; Lau et al, 2013; Broadbent, Galic and Stokes, 2013). It accurately supports the wellestablished three-factor structure of ASD (Hurst, Mitchell, Kimbrel, Kwapil and Nelson-Gray, 2007), which overlaps with the two-factor structure of DSM-5 (2013).

\section{Adult ADHD Self-Report Questionnaire v 1.1}

The Adult ADHD Self-Report Questionnaire v 1.1 is an 18-item questionnaire devised to assess ADHD traits (Kessler et al, 2005). It has a 5-point answer scale: Never, Rarely, Sometimes, Often, Very Often. Responses are then coded as scoring between 0 and 4. The first 6 questions are key in determining if the individual has ADHD traits and has been found to be most predictive of the condition. The questionnaire is separated into two subscales - inattentive and hyperactive - which each have nine questions. This questionnaire has consistently shown high convergent validity, correlation of subscales and test-retest reliability throughout different demographics and cultures (Silverstein et al, 2018; Evren et al, 2016).

\section{Procedure}

The study was conducted in accordance with University of Glasgow and Economic and Social Research Council (ESRC) ethical guidelines. Participants were introduced to VR via theBlu (WEVR, 2016). This game puts participants into an underwater scene. Participants cannot interact with the environment and spend around 5 minutes adapting to the surroundings and the virtual world. The Technical Proficiency Questionnaire was completed after this introduction. Short breaks are recommended for every VR experience. Due to the immersive quality of the tool it can sometimes disorient participants, particularly if they have not experienced VR prior to the experiment.

Participants were also introduced to the TiltBrush (Google, 2016) environment where they familiarised themselves with the functions of the controllers before proceeding to the first stage of the experiment. Participants were asked to draw the ROCF in the three-dimensional virtual environment. They could choose the environment they wished to draw in (e.g. space, blank colour, winter scene), as well as the colour and brush of the drawing tool. Once the ROCF image was presented to them, participants could move it anywhere they found fit. After the completion of the Copy condition, the ROCF image was removed from the environment and participants were asked to draw it from memory. 


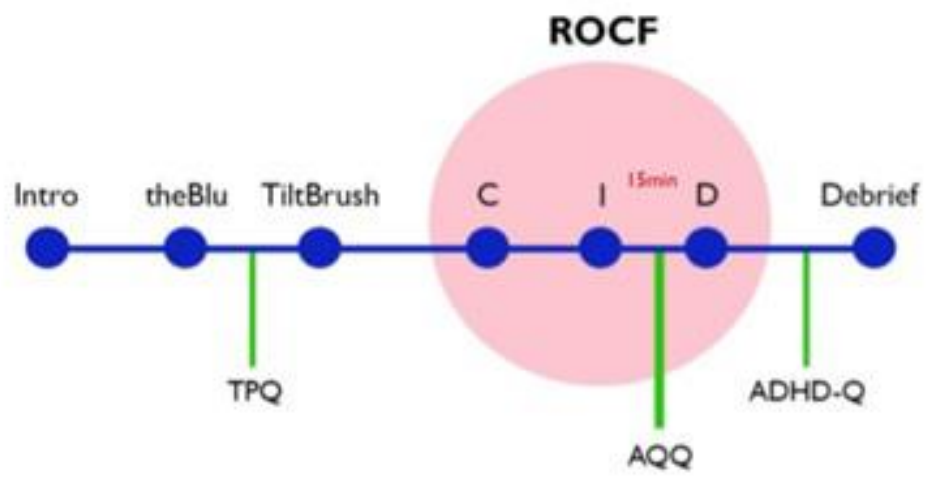

Figure 2. The experimental Procedure diagram used to ensure each participant followed the same protocol. The blue timeline shows the order of the procedure, where the pink circle indicates the experimental conditions of Rey-Osterrieth Complex Figure task: Copy, Immediate Recall and Delayed Recall (with 15min delay in between the two recall conditions). Green lines indicate questionnaires administered in the intervals between the elements of the experiment: TPQ - Technical Proficiency Questionnaire, AQQ - Autism Quotient Questionnaire, ADHD-Q - ADHD Questionnaire

The AQ was completed after the Immediate Recall condition, which resulted in 15 minutes break from the VR. The final task was to draw the ROCF from memory again. After the Delayed Recall participants were asked to fill in the ADHD questionnaire and were debriefed. The full experimental procedure is summarised in Figure 3.

An additional questionnaire was introduced after 32 participants were tested, so only 62 completed this questionnaire (see supplementary material for details). The majority of the first 32 participants tested expressed specific strategies they were using to remember the ROCF and, therefore, a decision was made to introduce this additional questionnaire. This questionnaire was introduced at the end of the Delayed Recall condition and before the debriefing.

\section{Data Analysis}

Perceptual scoring (Osterrieth, 1944) divides the ROCF into 18 elements and scores each one based on the placement of the element, distortion, completion and absence (Figure 4). The maximum score available is 36 where all elements are present, complete and accurate. See online supplementary materials for a detailed description of the perceptual scoring method. 


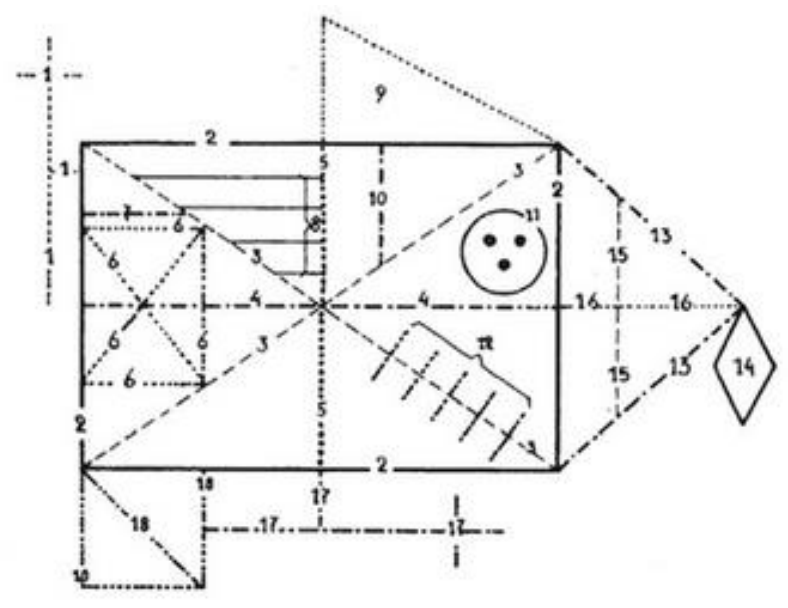

Figure 3. ROCF perceptual scoring system systemized by Paul-Alexandre Osterrieth in 1944. A detailed description of how each element was scored is available in online supplementary materials.

Organisational scoring (Hamby, Wilkins and Barry, 1993) scores the figure based on configural elements, secondary and detailed mistakes. If no configural mistakes are detected, the scorer checks for secondary mistakes. If no secondary mistakes detected, then the final step is checking mistakes in the details. Scores vary from 1 to 5 , where 1 is very poor organisation and 5 is excellent organisation (see online supplementary materials for a detailed description of the organisational scoring system used).

\section{Results}

The internal consistency as measured using Cronbach's alpha was very good for both the AQ ( $\alpha=$ $0.88)$ and $\mathrm{ADHD}$ questionnaires $(\alpha=0.91)$. The mean AQ scores of female participants (Mean $=$ $16.46, \mathrm{SD}=6.46$ ) was slightly lower than that of male participants (Mean $=18.50, \mathrm{SD}=12.13$ ). The opposite pattern was observed in ADHD questionnaire scores, where females (Mean $=2.23, \mathrm{SD}=$ 1.74) scored higher than males (Mean $=1.88, \mathrm{SD}=2.10$ ). The Spearman's Rho correlation between $\mathrm{ADHD}$ and AQ scores was insignificant, $\rho(60)=0.23, \mathrm{p}=0.07$. This relationship can be seen in Figure 5. The lack of significant relationship is in contrast to previous literature suggesting overlap between autistic and ADHD traits in the neurotypical population (Ronald et al, 2008). 


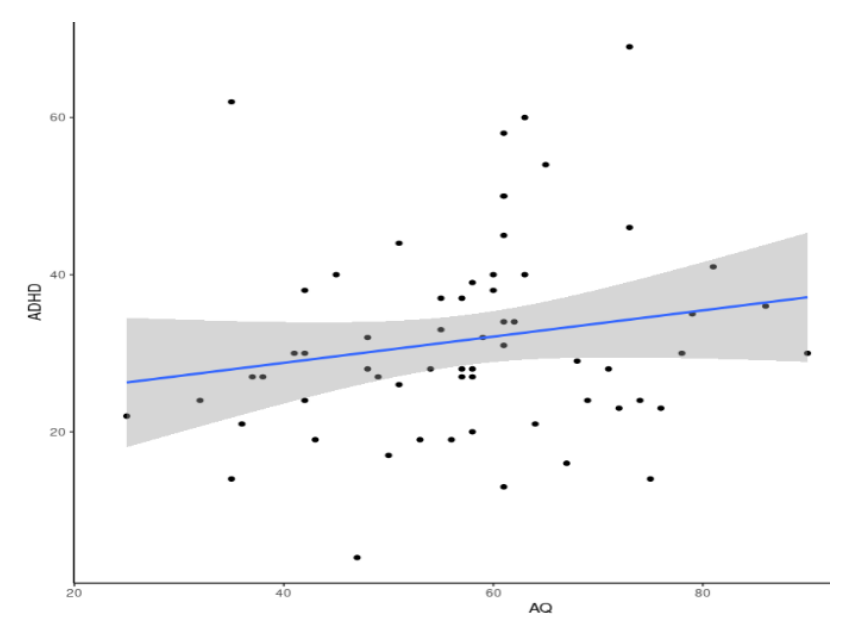

Figure 5. Scatterplot of AQ and ADHD scores.

ROCF perceptual scores for the Copy condition (Mean $=33.72, \mathrm{SD}=3.01$ ) were higher than for the Immediate Recall condition (Mean $=24,61, \mathrm{SD}=5.68$ ) and the Delayed Recall condition (Mean = $24.53, \mathrm{SD}=5.61)$. ROCF organisational scores showed the same trend with the Copy condition (Mean $=2.85, \mathrm{SD}=1.44)$ being higher than the Immediate Recall (Mean $=2.68, \mathrm{SD}=1.09)$ and the Delayed Recall $($ Mean $=2.66, \mathrm{SD}=1.09)$ conditions. These can be seen in Figure 7 .

A.

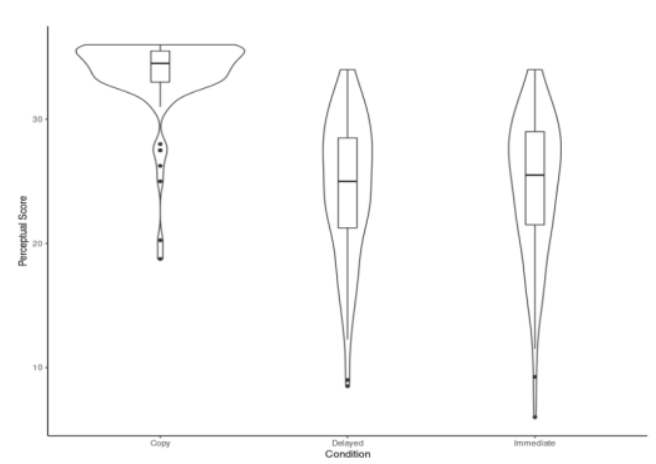

B.

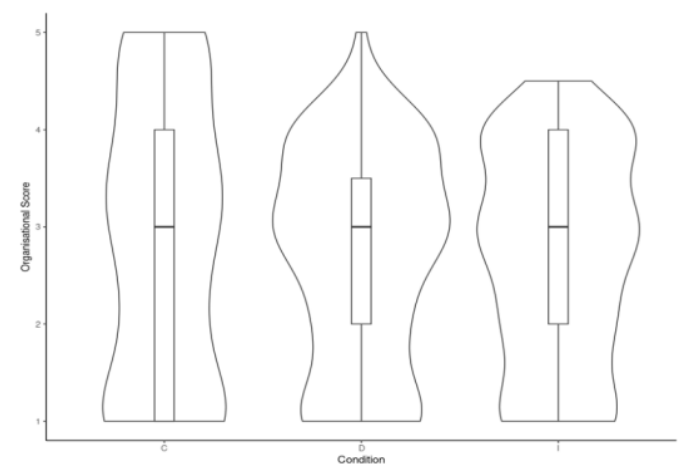

Figure 7. Visual comparison of ROCF scoring between copied, immediate recall, and delayed recall conditions. A. Perceptual scores; B. Organisational Scores.

To investigate the effects of the AQ and ADHD subscales on ROCF performance two multiple linear regression models were estimated for perceptual and organisational scores, the results of which are in Table 4 . The conditions were coded using backward difference encoding and all variables were mean centred and scaled. ADHD, perceptual, and organisational scores were transformed using Tukey's ladder of powers. The residuals of the final models were approximately normally distributed. The models were found to be significant for both perceptual score (adjusted $\mathrm{R}^{2}=0.53, \mathrm{~F}(9,176)=24.15$, $\mathrm{p}$ $<0.001$ ) and organisational score (adjusted $\mathrm{R}^{2}=0.08, \mathrm{~F}(9,176)=2.8, \mathrm{p}=0.004$ ). 
A.

\begin{tabular}{|l|l|l|l|l|}
\cline { 2 - 5 } \multicolumn{1}{c|}{} & $\beta$ Estimates & Standard Error & t-Statistic & $\mathrm{p}$-value \\
\hline Intercept & 0.10 & 0.05 & 2.05 & 0.041 \\
\hline Attention-to-detail & 0.23 & 0.06 & 4.11 & $<0.001$ \\
\hline Attention Switching & -0.06 & 0.07 & 0.82 & 0.411 \\
\hline Communication & 0.01 & 0.08 & 0.12 & 0.904 \\
\hline Imagination & -0.10 & 0.06 & 1.49 & 0.138 \\
\hline Social Skills & 0.14 & 0.08 & 1.68 & 0.095 \\
\hline Hyperactivity & -0.03 & 0.08 & 0.35 & 0.729 \\
\hline Inattentiveness & -0.04 & 0.08 & 0.51 & 0.611 \\
\hline Copy to Immediate Recall & -1.45 & 0.12 & 12.01 & $<0.001$ \\
\hline Immediate to Delayed Recall & -0.01 & 0.12 & 0.11 & 0.913 \\
\hline
\end{tabular}

B.

\begin{tabular}{|l|l|l|l|l|}
\cline { 2 - 5 } \multicolumn{1}{c|}{} & $\beta$ Estimates & Standard Error & t-Statistic & $\mathrm{p}$-value \\
\hline Intercept & 0.22 & 0.07 & 3.24 & 0.001 \\
\hline Attention-to-detail & 0.27 & 0.08 & 3.49 & $<0.001$ \\
\hline Attention Switching & -0.23 & 0.09 & 2.48 & 0.01 \\
\hline Communication & 0.07 & 0.11 & 0.63 & 0.528 \\
\hline Imagination & -0.19 & 0.09 & 2.18 & 0.031 \\
\hline Social Skills & 0.04 & 0.11 & 0.32 & 0.75 \\
\hline Hyperactivity & 0.15 & 0.11 & 1.36 & 0.149 \\
\hline Inattentiveness & -0.12 & 0.11 & 1.13 & 0.261 \\
\hline Copy to Immediate Recall & -0.30 & 0.17 & 1.80 & 0.073 \\
\hline Immediate to Delayed Recall & -0.07 & 0.17 & 0.40 & 0.692 \\
\hline
\end{tabular}

Table 4. Coefficients from multiple linear regressions of perceptual (A) and organisational (B) ROCF scores. Significant predictors are highlighted.

\section{Discussion}

The aim of the study was to investigate perceptual differences in local and global processing between participants with higher and lower levels of autistic and ADHD traits. We used Virtual Reality to present the Rey-Osterrieth Complex Figure task and provide a more immersive experience. Due to the exploratory nature of the study, we expected differences between the experimental conditions, however, we did not predict the direction of these differences. 
Contradictory to previous findings (Panagiotidi et al., 2019), autistic and ADHD traits were not related. The degree of overlap between autistic and ADHD traits appears particularly strong across communication and attention switching difficulties (Panagiotidi et al., 2019; Taylor, Charman \& Ronald, 2014). These traits are not always observed in University students; rather a higher expression of attention-to-detail is often reported (Kitazoe et al., 2017). Therefore, as our sample consisted primarily of university students, these inconsistent findings may be related to an overall limited presence of the relevant overlapping traits. Findings also indicated that variance in overall autistic and ADHD traits was not associated with visual processing styles. Recent research has proposed that individuals with ADHD may experience a local processing bias (Cohen \& Kalanthroff, 2019; Song \& Hakoda, 2015). This finding is not supported by the current study. As previously discussed, a number of ADHD traits are not always observed in University students (Kitazoe et al., 2017). Therefore, detecting significant changes in ROCF performance may not be possible in the current sample as relevant traits influencing visual processing preferences may not be present.

Furthermore, these results do not support the theories of Weak Central Coherence and Enhanced Perceptual Functioning that propose local bias in autistics. Rather, our findings demonstrate that those with a higher levels of autistic traits have global processing abilities that are similar to those with lower levels. Similarly, a review by Van der Hallen et al. (2015) reported that autistics show few significant reductions in global processing ability. This suggests that visual processing styles may not vary significantly when more autistic traits are expressed. Alternatively, visual processing styles may not be as identifiable within a general population as previously expected. Autism is extremely heterogenous and the sample used in this study may not provide an overall understanding into how symptoms vary across the spectrum. Across a general population, the average AQ score is approximately 17 (Ruzich et al., 2015) which was similar to our sample $(M=16.52)$. However, this sample was not fully representative of the broader autism spectrum as only one participant reached a score higher than 40 (remember the score for 32 or more might be linked to diagnosable ASD). As a result, further research would benefit from investigating a sample with higher AQ scores to determine whether differences in visual processing styles can be detected in those expressing a high number of autistic traits. This is expected to provide useful insight into how perceptual differences vary across a broader continuum of expressed autistic traits.

Interestingly, the AQ attention-to-detail subscale was associated with performance in the recall conditions for perceptual and organisational ROCF scores. This is a new finding that suggests attention-to-detail may be related to more accurate recall of local information. The AQ attention-todetail subscale was predictive of higher organisational scores. This indicates a more detail-focused cognitive style may support the processing of local information. This finding has potentially important implications for understanding the perceptual experience of autistics. First, this suggests that 
attention-to-detail may be a key autistic trait related to visual processing. Recent research has begun to study attention-to-detail in relation to visual processing that support this idea. For example,

Burghoorn et al. (2020) identified that individuals scoring higher on AQ attention-to-detail demonstrated a bias towards local visual perception when performing the Embedded Figures Task. Therefore, this suggests those scoring higher in autistic traits may have a tendency to locate local information. Further, previous research has identified differences between AQ attention-to-detail and autistic traits in eye tracking tasks related to facial recognition (Davis et al., 2017). Specifically, it was found that individuals with a higher attention-to-detail score were more likely to look at the eyes of a face than those who scored higher on social traits. This suggests that the subscales of the AQ are fairly distinct and play a role in different aspects of executive functioning and information processing. Therefore, the collective study of the AQ subscales may provide an explanation as to why such contradictory findings discussing visual processing styles are present in the current literature (see Millington \& Simmons, 2021).

The Attention Switching and Imagination subscales of the AQ were also found to be predictors in how well participants performed as measured on the organisational scale. Previous research has linked the Attention Switching subscale to autism diagnosis in children (Hatta, et al., 2019) and broader executive function differences (Tran, Arredondo \& Yoshida, 2018). Organisational scores were not linked to ADHD traits in our sample, including the Hyperactivity and Inattentiveness subscales. This might suggest that higher autistic traits are linked to a different cognitive pattern than we initially anticipated, and higher ADHD traits might not have any effect. This is further supported by the link between organisational scores and Imagination subscale of the AQ. Imagination has been previously linked to creativity and cognitive flexibility (Roberts et al, 2017; Laureiro-Martinez et al, 2018), however, autism research often suggests that autistic traits are linked to limited imagination and social skills (Dance et al, 2021). Our findings challenge the notion that autistic creativity is somewhat limited and relates to literature suggesting that autistic individuals have detail-focused cognitive processing and drawing styles (Darewych, Newton \& Farrugie, 2018) and this unexpected relationship between cognitive flexibility and visual processing should be explored further.

\section{Limitations and Future directions}

The novelty of the task provides us with a quantifiable measure of the three-dimensional sketches drawn in TiltBrush. However, it is unclear how it can be used to understand individual differences between the participants and their behaviour yet. The data does not show any differences between the conditions and the measured traits (AQ and ADHD) in the current experiment. This might suggest that the task itself is not suitable. Completion time is the longest for the Copy condition suggesting that it takes a while to process the ROCF. 
In addition, the ROCF is a line drawing presented to the participants as a two-dimensional object. A three-dimensional equivalent of the figure might be easier to process in the immersive VR environment. The majority of the participants were not familiar with the Rey-Osterrieth Complex Figure and took longer to familiarise themselves with it, which is evident from longer completion times in the Copy condition. Running the standard two-dimensional ROCF task alongside the new three-dimensional version might illuminate parts of the task which are most challenging. Moreover, the exposure to the figure in a familiar 'paper-and-pencil' environment might help participants process information in VR more efficiently.

A

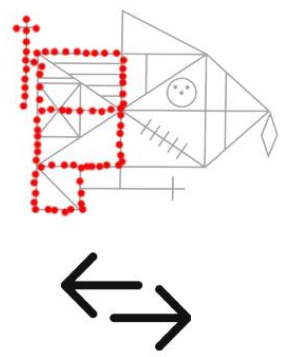

B

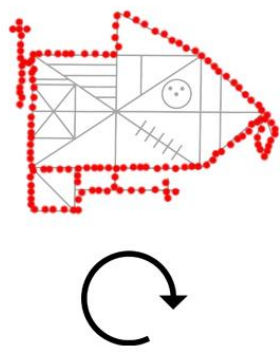

C

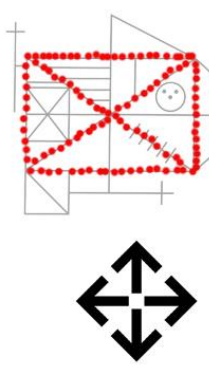

Figure 8. Proposed drawing styles. A - left to right (or right to left); B - circular; C - global elements defined by standard ROCF scoring systems

Individual differences were evident in the data and a more thorough qualitative analysis might reveal the distinct ways in which participants are approaching the task. Figure 8 illustrates just a few strategies adapted by the participants, where some prefer the standard global drawing style (as defined by Rey (1941) and Osterrieth (1944)) and others adapt alternative strategies of drawing (left to right or in a circular fashion). These alternative drawing styles would affect both perceptual and organisation ROCF scoring and could be a contributing factor to the lack of significant results. This unexpected behaviour in our participants could also be linked to the significance of the Imagination subscale of the AQ as a predictor of organisational scores.

TiltBrush from Google was used to present the experiment and record the data. Only one of the participants was left-handed and it is evident that they took longer to draw and had multiple technical issues. TiltBrush is currently unable to adapt to left-handed participants. This does not mean that lefthanded individuals cannot draw in VR, however, it can often be difficult to navigate the threedimensional environment, select tools and draw accurately. Recently, Tiltbrush has been made open source material, which means that the app can be modified. Future work could look into simplifying the app and amending it to suit experimental visual processing tasks. 


\section{Conclusion}

Virtual Reality has proved itself to be a fruitful new source of information. The experiments reported here are an example of how classic psychological paradigms can be transferred into the virtual world. Our novel method of data extraction provides us with extensive information on how individuals navigate themselves in the virtual world. Further investigation of the distinct individual preferences in drawing tasks in VR could lead to a better understanding on how we process visuospatial information. As a result, such findings would inevitably extend to industrial applications. 


\section{References}

Abu-Akel, A., Allison, C., Baron-Cohen, S., \& Heinke, D. (2019). The distribution of autistic traits across the autism spectrum: evidence for discontinuous dimensional subpopulations underlying the autism continuum. Molecular autism, 10(1), 1-13.

Akshoomoff, N. A., \& Stiles, J. (1995). Developmental trends in visuospatial analysis and planning: I. Copying a complex figure. Neuropsychology, 9(3), 364.

Akshoomoff, N. A., \& Stiles, J. (1995). Developmental trends in visuospatial analysis and planning: II. Memory for a complex figure. Neuropsychology, 9(3), 378.

American Psychiatric Association. (2013). Diagnostic and statistical manual of mental disorders (5th ed.). Arlington, VA: American Psychiatric Publishing.

Antshel, K. M., Hier, B. O., \& Barkley, R. A. (2014). Executive functioning theory and ADHD. In Handbook of executive functioning (pp. 107-120). Springer, New York, NY.

Baird, G., Simonoff, E., Pickles, A., Chandler, S., Loucas, T., Meldrum, D., \& Charman, T. (2006). Prevalence of disorders of the autism spectrum in a population cohort of children in South Thames: the Special Needs and Autism Project (SNAP). The lancet, 368(9531), 210-215.

Barkley, R. A., Grodzinsky, G., \& DuPaul, G. J. (1992). Frontal lobe functions in attention deficit disorder with and without hyperactivity: A review and research report. Journal of abnormal child psychology, 20(2), 163-188.

Baron-Cohen, S., Wheelwright, S., Skinner, R., Martin, J., \& Clubley, E. (2001). The autism-spectrum quotient (AQ): Evidence from asperger syndrome/high-functioning autism, malesand females, scientists and mathematicians. Journal of autism and developmental disorders, 31(1), 5-17.

Beebe, D. W., DiFrancesco, M. W., Tlustos, S. J., McNally, K. A., \& Holland, S. K. (2009). Preliminary fMRI findings in experimentally sleep-restricted adolescents engaged in a working memory task. Behavioral and Brain Functions, 5(1), 1-7.

Bellani, M., Fornasari, L., Chittaro, L., \& Brambilla, P. (2011). Virtual reality in autism: state of the art. Epidemiology and psychiatric sciences, 20(3), 235-238.

Bogdashina, O. (2005). Theory of Mind and the Triad of Perspectives on Autism and Asperger Syndrome: A view from the bridge. Jessica Kingsley Publishers.

Bogdashina, O. (2016). Sensory perceptual issues in autism and asperger syndrome: different sensory experiences-different perceptual worlds. Jessica Kingsley Publishers.

Broadbent, J., Galic, I., \& Stokes, M. A. (2013). Validation of autism spectrum quotient adult version in an Australian sample. Autism research and treatment, 2013.

Brown, L., Millington, E., Robertson, A. E., \& Simmons, D. (2021). Children's Glasgow Sensory Questionnaire (C-GSQ): Validation of a Simplified and Visually Aided Questionnaire. PsyArXiv. April, 27.

Brugha, T. S., McManus, S., Bankart, J., Scott, F., Purdon, S., Smith, J., ... \& Meltzer, H. (2011). Epidemiology of autism spectrum disorders in adults in the community in England. Archives of general psychiatry, 68(5), 459-465. 
Bury, S. M., Jellett, R., Spoor, J. R., \& Hedley, D. (2020). "It defines who I am" or "It's something I have": What language do [autistic] Australian adults [on the autism spectrum] prefer?. Journal of autism and developmental disorders, 1-11.

Chen, S. F., Chien, Y. L., Wu, C. T., Shang, C. Y., Wu, Y. Y., \& Gau, S. S. (2016). Deficits in executive functions among youths with autism spectrum disorders: an age-stratified analysis. Psychological medicine, 46(8), 1625-1638.

Cohen, E., \& Kalanthroff, E. (2019). Visuospatial processing bias in ADHD: A potential artifact in the wechsler adult intelligence scale and the rorschach inkblots test. Psychological Assessment, 31(5), 699-706

Cribb, S. J., Olaithe, M., Di Lorenzo, R., Dunlop, P. D., \& Maybery, M. T. (2016). Embedded figures test performance in the broader autism phenotype: a meta-analysis. Journal of Autism and Developmental Disorders, 46(9), 2924-2939.

Dance, C. J., Jaquiery, M., Eagleman, D. M., Porteous, D., Zeman, A., \& Simner, J. (2021). What is the relationship between Aphantasia, Synaesthesia and Autism?. Consciousness and Cognition, 89, 103087.

Darewych, O. H., Newton, N. J., \& Farrugie, K. W. (2018). Investigating Imagination in Adults With Autism Spectrum Disorder With Art. Based Assessments. Journal on Developmental Disabilities, 23(2), $27-$ 36.

Davis, J., McKone, E., Zirnsak, M., Moore, T., O'Kearney, R., Apthorp, D., \& Palermo, R. (2017). Social and attention-to-detail subclusters of autistic traits differentially predict looking at eyes and face identity recognition ability. British Journal of Psychology, 108(1), 191-219.

Dredge, Stuart (2014). Facebook closes its \$2bn Oculus Rift acquisition. What next?. Guardian.

Elias, Z. M., Batumalai, U. M., \& Azmi, A. N. H. (2019). Virtual reality games on accommodation and convergence. Applied ergonomics, 81, 102879.

Evren, C., Umut, G., Bozkurt, M., Unal, G. T., Agachanli, R., \& Evren, B. (2016). Psychometric properties of the Turkish version of the Adult ADHD Self-Report Scale (ASRS-v1. 1) in a sample of Inpatients with alcohol use disorder. Dusunen Adam The Journal of Psychiatry and Neurological Sciences, 29(2), 109.

Fleischmann, A., \& Fleischmann, C. (2012). Carly's voice: Breaking through autism. Simon and Schuster.

Fletcher-Watson, S., \& Happé, F. (2019). Autism: A new introduction to psychological theory and current debate. Routledge.

Freeseman, L. J., Colombo, J., \& Coldren, J. T. (1993). Individual differences in infant visual attention: Fourmonth-olds' discrimination and generalization of global and local stimulus properties. Child Development, 64(4), 1191-1203.

Frith, U. (1989). Autism and "theory of mind". In Diagnosis and treatment of autism (pp. 33-52). Springer, Boston, MA.

Frith, U. (2003). Autism: Explaining the enigma. Blackwell Publishing.

Gasper, K., \& Clore, G. L. (2002). Attending to the big picture: Mood and global versus local processing of visual information. Psychological science, 13(1), 34-40.

Goldstein, E. B., \& Brockmole, J. (2016). Sensation and perception. Cengage Learning. 
Grandin, T., \& Duffy, K. (2008). Developing talents: Careers for individuals with Asperger syndrome and high-functioning autism. AAPC Publishing.

Groom, M. J., Kochhar, P., Hamilton, A., Liddle, E. B., Simeou, M., \& Hollis, C. (2017). Atypical processing of gaze cues and faces explains comorbidity between autism spectrum disorder (ASD) and attention deficit/hyperactivity disorder (ADHD). Journal of autism and developmental disorders, 47(5), 14961509.

Hamby, S. L., Wilkins, J. W., \& Barry, N. S. (1993). Organizational quality on the Rey-Osterrieth and Taylor Complex Figure Tests: A new scoring system. Psychological assessment, 5(1), 27.

Happé, F. G., \& Booth, R. D. (2008). The power of the positive: Revisiting weak coherence in autism spectrum disorders. Quarterly journal of experimental psychology, 61(1), 50-63.

Happé, F., \& Frith, U. (1996). The neuropsychology of autism. Brain, 119(4), 1377-1400.

Happé, F., \& Ronald, A. (2008). The 'fractionable autism triad': a review of evidence from behavioural, genetic, cognitive and neural research. Neuropsychology review, 18(4), 287-304.

Hatta, K., Hosozawa, M., Tanaka, K., \& Shimizu, T. (2019). Exploring Traits of Autism and Their Impact on Functional Disability in Children with Somatic Symptom Disorder. Journal of autism and developmental disorders, 49(2), 729-737.

Hill, M. (2011). Young Children and Their Perceptions of Colour: An Exploratory Study.

Hoffman, E. J. (2009). Clinical features and diagnosis of autism and other pervasive developmental disorders. Primary Psychiatry, 16(1).

Hurst, R. M., Mitchell, J. T., Kimbrel, N. A., Kwapil, T. K., \& Nelson-Gray, R. O. (2007). Examination of the reliability and factor structure of the Autism Spectrum Quotient (AQ) in a non-clinical sample. Personality and Individual Differences, 43(7), 1938-1949.

Hutsler, J. J., \& Casanova, M. F. (2016). Cortical construction in autism spectrum disorder: columns, connectivity and the subplate. Neuropathology and applied neurobiology, 42(2), 115-134.

Johnson, S. C., Lowery, N., Kohler, C., \& Turetsky, B. I. (2005). Global-local visual processing in schizophrenia: evidence for an early visual processing deficit. Biological psychiatry, 58(12), 937-946.

Kalanthroff, E., Naparstek, S., \& Henik, A. (2013). Spatial processing in adults with attention deficit hyperactivity disorder. Neuropsychology, 27(5), 546.

Kandalaft, M. R., Didehbani, N., Krawczyk, D. C., Allen, T. T., \& Chapman, S. B. (2013). Virtual reality social cognition training for young adults with high-functioning autism. Journal of autism and developmental disorders, 43(1), 34-44.

Ke, F., \& Im, T. (2013). Virtual-reality-based social interaction training for children with high-functioning autism. The Journal of Educational Research, 106(6), 441-461.

Kessler, R. C., Adler, L., Ames, M., Demler, O., Faraone, S., Hiripi, E. V. A., ... \& Ustun, T. B. (2005). The World Health Organization Adult ADHD Self-Report Scale (ASRS): a short screening scale for use in the general population. Psychological medicine, 35(2), 245-256.

Kiselev, S. (2019). F57. Children With ADHD Have Deficit in Reproducing the Rey-Osterrieth Complex Figure in Delayed Recall Condition. Biological Psychiatry, 85(10), S235. 
Kitazoe, N., Fujita, N., Izumoto, Y., Terada, S. I., \& Hatakenaka, Y. (2017). Whether the autism spectrum quotient consists of two different subgroups? Cluster analysis of the autism spectrum quotient in general population. Autism, 21(3), 323-332.

Kuriakose, S., \& Lahiri, U. (2016). Design of a physiology-sensitive VR-based social communication platform for children with autism. IEEE Transactions on Neural Systems and Rehabilitation Engineering, 25(8), 1180-1191.

Kuschner, E. S., Bodner, K. E., \& Minshew, N. J. (2009). Local vs. global approaches to reproducing the Rey Osterrieth complex figure by children, adolescents, and adults with high-functioning autism. Autism Research, 2(6), 348-358.

Laffont, P. Y., \& Hasnain, A. (2017). Adaptive dynamic refocusing: toward solving discomfort in virtual reality. In ACM SIGGRAPH 2017 Emerging Technologies (pp. 1-2).

Lai, C. L. E., Lau, Z., Lui, S. S., Lok, E., Tam, V., Chan, Q., ... \& Cheung, E. F. (2017). Meta-analysis of neuropsychological measures of executive functioning in children and adolescents with highfunctioning autism spectrum disorder. Autism Research, 10(5), 911-939.

Lang, K., Roberts, M., Harrison, A., Lopez, C., Goddard, E., Khondoker, M., ... \& Tchanturia, K. (2016). Central coherence in eating disorders: a synthesis of studies using the Rey Osterrieth complex figure test. PloS one, 11(11), e0165467.

Lau, W. Y. P., Gau, S. S. F., Chiu, Y. N., Wu, Y. Y., Chou, W. J., Liu, S. K., \& Chou, M. C. (2013). Psychometric properties of the Chinese version of the Autism Spectrum Quotient (AQ). Research in developmental disabilities, 34(1), 294-305.

Laureiro-Martínez, D., \& Brusoni, S. (2018). Cognitive flexibility and adaptive decision-making: Evidence from a laboratory study of expert decision makers. Strategic Management Journal, 39(4), 1031-1058.

Li, W., Lai, T. M., Bohon, C., Loo, S. K., McCurdy, D., Strober, M., ... \& Feusner, J. (2015). Anorexia nervosa and body dysmorphic disorder are associated with abnormalities in processing visual information. Psychological medicine, 45(10), 2111-2122.

Lichtenstein, P., Carlström, E., Råstam, M., Gillberg, C., \& Anckarsäter, H. (2010). The genetics of autism spectrum disorders and related neuropsychiatric disorders in childhood. American Journal of Psychiatry, 167(11), 1357-1363.

Lopez, C., Tchanturia, K., Stahl, D., \& Treasure, J. (2008). Central coherence in eating disorders: a systematic review. Psychological medicine, 38(10), 1393-1404.

Lubke, G. H., Hudziak, J. J., Derks, E. M., van Bijsterveldt, T. C., \& Boomsma, D. I. (2009).

Luna, B., Doll, S. K., Hegedus, S. J., Minshew, N. J., \& Sweeney, J. A. (2007). Maturation of executive function in autism. Biological psychiatry, 61(4), 474-481.

Maskey, M., Lowry, J., Rodgers, J., McConachie, H., \& Parr, J. R. (2014). Reducing specific phobia/fear in young people with autism spectrum disorders (ASDs) through a virtual reality environment intervention. PloS one, 9(7), e100374.

Maternal ratings of attention problems in ADHD: evidence for the existence of a continuum. Journal of the American Academy of Child \& Adolescent Psychiatry, 48(11), 1085-1093. 
Matsentidou, S., \& Poullis, C. (2014, January). Immersive visualizations in a VR cave environment for the training and enhancement of social skills for children with autism. In 2014 International Conference on Computer Vision Theory and Applications (VISAPP) (Vol. 3, pp. 230-236). IEEE.

Mcconley, R., Martin, R., BaÑos, J., Blanton, P., \& Faught, E. (2006). Global/local scoring modifications for the Rey-Osterrieth Complex Figure: Relation to unilateral temporal lobe epilepsy patients. Journal of the International Neuropsychological Society, 12(3), 383-390.

McKone, E., Davies, A. A., Fernando, D., Aalders, R., Leung, H., Wickramariyaratne, T., \& Platow, M. J. (2010). Asia has the global advantage: Race and visual attention. Vision research, 50(16), 1540-1549.

Melrose, R. J., Harwood, D., Khoo, T., Mandelkern, M., \& Sultzer, D. L. (2013). Association between cerebral metabolism and Rey-Osterrieth Complex Figure Test performance in Alzheimer's disease. Journal of clinical and experimental neuropsychology, 35(3), 246-258.

Miller, M., \& Hinshaw, S. P. (2010). Does childhood executive function predict adolescent functional outcomes in girls with ADHD?. Journal of abnormal child psychology, 38(3), 315-326.

Minshew, N. J., Goldstein, G., Taylor, H. G., \& Siegel, D. J. (1994). Academic achievement in high functioning autistic individuals. Journal of Clinical and Experimental Neuropsychology, 16(2), 261270.

Minshew, N. J., Muenz, L. R., Goldstein, G., \& Payton, J. B. (1992). Neuropsychological functioning in nonmentally retarded autistic individuals. Journal of Clinical and Experimental Neuropsychology, 14(5), 749-761.

Moritz, S., \& Wendt, M. (2006). Processing of local and global visual features in obsessive-compulsive disorder. Journal of the International Neuropsychological Society, 12(4), 566-569.

Mottron, L., \& Burack, J. A. (2001). Enhanced perceptual functioning in the development of autism.

Mottron, L., Dawson, M., Soulieres, I., Hubert, B., \& Burack, J. (2006). Enhanced perceptual functioning in autism: an update, and eight principles of autistic perception. Journal of autism and developmental disorders, 36(1), 27-43.

Muth, A., Hönekopp, J., \& Falter, C. M. (2014). Visuo-spatial performance in autism: a metaanalysis. Journal of Autism and Developmental Disorders, 44(12), 3245-3263.

Osterrieth, P. A. (1944). Le test de copie d'une figure complexe; contribution a l'etude de la perception et de la memoire. Archives de psychologie.

Ozonoff, S., Pennington, B. F., \& Rogers, S. J. (1991). Executive function deficits in high-functioning autistic individuals: relationship to theory of mind. Journal of child Psychology and Psychiatry, 32(7), 1081 1105.

Padmanaban, N., Konrad, R., Stramer, T., Cooper, E. A., \& Wetzstein, G. (2017). Optimizing virtual reality for all users through gaze-contingent and adaptive focus displays. Proceedings of the National Academy of Sciences, 114(9), 2183-2188.

Panagiotidi, M., Overton, P. G., \& Stafford, T. (2018). The relationship between ADHD traits and sensory sensitivity in the general population. Comprehensive psychiatry, 80, 179-185.

Panagiotidi, M., Overton, P. G., \& Stafford, T. (2019). Co-occurrence of ASD and ADHD traits in an adult population. Journal of attention disorders, 23(12), 1407-1415.

Parsons, S. (2016). Authenticity in Virtual Reality for assessment and intervention in autism: A conceptual review. Educational Research Review, 19, 138-157. 
Paulus, M. M., Straube, A., \& Eggert, T. (2017). Vergence-accommodation conflict in virtual reality displays induces phoria adaptation. Journal of neurology, 264(1), 16-17.

Rey, A. (1941). L'examen psychologique dans les cas d'encéphalopathie traumatique.(Les problems.). Archives de psychologie.

Roalf, D., Lowery, N., \& Turetsky, B. I. (2006). Behavioral and physiological findings of gender differences in global-local visual processing. Brain and Cognition, 60(1), 32-42.

Roberts, R. P., Wiebels, K., Sumner, R. L., van Mulukom, V., Grady, C. L., Schacter, D. L., \& Addis, D. R. (2017). An fMRI investigation of the relationship between future imagination and cognitive flexibility. Neuropsychologia, 95, 156-172.

Robertson, A. E., \& Simmons, D. R. (2013). The relationship between sensory sensitivity and autistic traits in the general population. Journal of Autism and Developmental disorders, 43(4), 775-784.

Robertson, A. E., \& Simmons, D. R. (2015). The sensory experiences of adults with autism spectrum disorder: A qualitative analysis. Perception, 44(5), 569-586.

Rommelse, N. N., Geurts, H. M., Franke, B., Buitelaar, J. K., \& Hartman, C. A. (2011). A review on cognitive and brain endophenotypes that may be common in autism spectrum disorder and attentiondeficit/hyperactivity disorder and facilitate the search for pleiotropic genes. Neuroscience \& Biobehavioral Reviews, 35(6), 1363-1396.

Ronald, A., Simonoff, E., Kuntsi, J., Asherson, P., \& Plomin, R. (2008). Evidence for overlapping genetic influences on autistic and ADHD behaviours in a community twin sample. Journal of Child psychology and Psychiatry, 49(5), 535-542.

Ruzich, E., Allison, C., Smith, P., Watson, P., Auyeung, B., Ring, H., \& Baron-Cohen, S. (2015). Measuring autistic traits in the general population: a systematic review of the Autism-Spectrum Quotient (AQ) in a nonclinical population sample of 6,900 typical adult males and females. Molecular autism, 6(1), 112.

Sadler-Smith, E. (2011). The intuitive style: Relationships with local/global and verbal/visual styles, gender, and superstitious reasoning. Learning and Individual Differences, 21(3), 263-270.

Savickaite, S., McDonnell, N. \& Simmons, D. R. (2021). Definitions in Virtual Reality: a literature review focusing on autism research. Computers in Human Behaviour [under review].

Schauder, K. B., \& Bennetto, L. (2016). Toward an interdisciplinary understanding of sensory dysfunction in autism spectrum disorder: an integration of the neural and symptom literatures. Frontiers in neuroscience, 10, 268.

Shah, A., \& Frith, U. (1983). An islet of ability in autistic children: A research note. Journal of child Psychology and Psychiatry, 24(4), 613-620.

Shah, A., \& Frith, U. (1993). Why do autistic individuals show superior performance on the block design task?. Journal of Child Psychology and Psychiatry, 34(8), 1351-1364.

Shin, N. Y., Lee, T. Y., Kim, E., \& Kwon, J. S. (2014). Cognitive functioning in obsessive-compulsive disorder: a meta-analysis. Psychological medicine, 44(6), 1121-1130.

Shorr, J. S., Delis, D. C., \& Massman, P. J. (1992). Memory for the Rey-Osterrieth Figure: Perceptual clustering, encoding, and storage. Neuropsychology, 6(1), 43. 
Silverstein, M. J., Alperin, S., Faraone, S. V., Kessler, R. C., \& Adler, L. A. (2018). Test-retest reliability of the adult ADHD Self-Report Scale (ASRS) v1. 1 Screener in non-ADHD controls from a primary care physician practice. Family practice, 35(3), 336-341.

Simmons, D. R., \& Todorova, G. K. (2018). Local versus global processing in autism: Special section editorial.

Simmons, D. R., Robertson, A. E., McKay, L. S., Toal, E., McAleer, P., \& Pollick, F. E. (2009). Vision in autism spectrum disorders. Vision research, 49(22), 2705-2739.

Smith, M. J., Ginger, E. J., Wright, K., Wright, M. A., Taylor, J. L., Humm, L. B., ... \& Fleming, M. F. (2014). Virtual reality job interview training in adults with autism spectrum disorder. Journal of autism and developmental disorders, 44(10), 2450-2463.

Song, Y., \& Hakoda, Y. (2018). Selective impairment of basic emotion recognition in people with autism: Discrimination thresholds for recognition of facial expressions of varying intensities. Journal of autism and developmental disorders, 48(6), 1886-1894.

Steam (2003). Valve Corporation [Computer Software].

Stevenson, J. L., \& Hart, K. R. (2017). Psychometric properties of the autism-spectrum quotient for assessing low and high levels of autistic traits in college students. Journal of Autism and Developmental Disorders, 47(6), 1838-1853.

Taylor, M. J., Charman, T., \& Ronald, A. (2015). Where are the strongest associations between autistic traits and traits of ADHD? Evidence from a community-based twin study. European child \& adolescent psychiatry, 24(9), 1129-1138.

theBlu (2016). WEVR [Computer Software].

TiltBrush (2016). Google [Computer Software].

Tran, C. D., Arredondo, M. M., \& Yoshida, H. (2019). Early executive function: The influence of culture and bilingualism. Bilingualism: Language and Cognition, 22(4), 714-732.

Tsatsanis, K. D., Noens, I. L., Illmann, C. L., Pauls, D. L., Volkmar, F. R., Schultz, R. T., \& Klin, A. (2011). Managing complexity: Impact of organization and processing style on nonverbal memory in autism spectrum disorders. Journal of Autism and Developmental Disorders, 41(2), 135-147.

Van der Hallen, R., Evers, K., Brewaeys, K., Van den Noortgate, W., \& Wagemans, J. (2015). Global processing takes time: A meta-analysis on local-global visual processing in ASD. Psychological bulletin, 141(3), 549.

Van Eylen, L., Boets, B., Steyaert, J., Wagemans, J., \& Noens, I. (2018). Local and global visual processing in autism spectrum disorders: Influence of task and sample characteristics and relation to symptom severity. Journal of Autism and Developmental Disorders, 48(4), 1359-1381.

Waber, D. P., \& Bernstein, J. H. (1995). Performance of learning-disabled and non-learning-disabled children on the Rey-Osterrieth Complex Figure: Validation of the developmental scoring system. Developmental neuropsychology, 11(2), 237-252.

Wagemans, J., Elder, J. H., Kubovy, M., Palmer, S. E., Peterson, M. A., Singh, M., \& von der Heydt, R. (2012). A century of Gestalt psychology in visual perception: I. Perceptual grouping and figureground organization. Psychological bulletin, 138(6), 1172.

Wang, M., \& Reid, D. (2011). Virtual reality in pediatric neurorehabilitation: attention deficit hyperactivity disorder, autism and cerebral palsy. Neuroepidemiology, 36(1), 2-18. 
Watanabe, K., Ogino, T., Nakano, K., Hattori, J., Kado, Y., Sanada, S., \& Ohtsuka, Y. (2005). The ReyOsterrieth Complex Figure as a measure of executive function in childhood. Brain and Development, 27(8), 564-569.

Weber, R. C., Riccio, C. A., \& Cohen, M. J. (2013). Does Rey Complex Figure copy performance measure executive function in children?. Applied Neuropsychology: Child, 2(1), 6-12.

Wilson, N. A., \& Batchelor, J. (2015). Examining Rey Complex Figure Test organization in healthy adults. Journal of clinical and experimental neuropsychology, 37(10), 1052-1061.

Yovel, I., Revelle, W., \& Mineka, S. (2005). Who sees trees before forest? The obsessive-compulsive style of visual attention. Psychological science, 16(2), 123-129. 